\title{
Equinodermos del Museo de Zoología de la Universidad de Costa Rica
}

\author{
Juan José Alvarado ${ }^{1,2,3}$, José Leonardo Chacón-Monge ${ }^{2,3}$, Francisco Alonso Solís-Marín ${ }^{4}$, \\ Tania Pineda-Enríquez ${ }^{4,5}$, Andrea Alejandra Caballero-Ochoa ${ }^{4}$, Sofía Solano Rivera ${ }^{2,3}$ \\ \& Raquel Romero Chaves ${ }^{1}$ \\ 1. Centro de Investigación en Ciencias del Mar y Limnología, Universidad de Costa Rica; juan.alvarado@ucr.ac.cr \\ 2. Escuela de Biología, Universidad de Costa Rica; raquelromerochaves@gmail.com \\ 3. Museo de Zoología, Universidad de Costa Rica; 1.cuacser@gmail.com; sofisr.56@gmail.com \\ 4. Escuela de Biología, Facultad de Ciencias, Universidad Nacional Autónoma de México (UNAM), Circuito Exterior \\ s/n, Ciudad Universitaria, Apdo. 70-305, Ciudad de México, México, C.P. 04510; \\ a.caballero.ochoa@ciencias.unam.mx \\ 5. Department of Zoology, Division of Invertebrate Zoology, Florida Museum of Natural History, University of Florida, \\ Gainesville; pinedae@ufl.edu; tpinedae@gmail.com
}

Recibido 24-I-2017. Corregido 20-III-2017. Aceptado 02-V-2017.

\begin{abstract}
Echinoderms from the Museum of Zoology from the Universidad de Costa Rica. The Museum of Zoology, Universidad de Costa Rica (MZUCR) was founded in 1966 and houses the most complete collection of vertebrates and invertebrates in Costa Rica. The MZUCR currently has 24 collections containing more than five million specimens, and more than 13000 species. The earliest collections date back to 1960 and include fishes, reptiles, amphibians, polychaetes, crustaceans and echinoderms. For the latter group, the MZUCR has a total of 157 species, in 1173 lots and 4316 specimens. These 157 species represent $54 \%$ of the total species of echinoderms from Costa Rica. The remaining species are distributed in the following institutions: California Academy of Sciences (CAS) (4.8\%), Scripps Oceanographic Institute (SIO) (5.2\%), National Echinoderm Collection "Dr. Ma. Elena Caso" from the National Autonomous University of Mexico (ICML-UNAM) (12.7\%), the National Museum of Natural History, Smithsonian Institute (USNM) (35.1\%), and the Harvard Museum of Comparative Zoology (19.2\%). There may be material from Costa Rica in the Natural History Museum of Denmark (NCD) and the Natural History Museum of Los Angeles (LACM), however, there was no access to such collections. There are $9.6 \%$ that do not appear in museums, but are reported in the literature. Based on this revision, the taxonomic list of echinoderms for Costa Rica is updated to 293 species, 152 genera, 75 families, 30 orders and 5 classes. The Pacific coast of Costa Rica has 153 species, followed by the Isla del Coco with 134 and the Caribbean coast with 65. Holothuria is the most diverse genus with 25 species. Rev. Biol. Trop. 65(Suppl. 1): S272-S287. Epub 2017 November 01.
\end{abstract}

Key words: zoological collections; sampling effort; high diversity spots; research gaps; new reports.

Los museos de colecciones biológicas son herramientas fundamentales para comprender en mayor detalle la vida en la Tierra, al documentar la biodiversidad y su distribución, se convierten en referentes para la educación e investigación (Ponder, Carter, Flemons, \& Chapman, 2001; Winker, 2004). Estas colecciones pueden contribuir a una gran gama de estudios como análisis biogeográficos, evolutivos, cambios en la composición de una comunidad en el presente como a nivel geológico (muestras de fósiles), genéticos, bioquímicos, isotópicos, y de elementos trazas (Lane, 1996; Shaffer, Fisher, \& Davidson, 1998; Suarez \& Tsutsui, 2004; Lister \& Climate Change Research Group, 2011). En este sentido, los museos que albergan colecciones biológicas poseen información crucial para la toma de decisiones de la conservación de la biodiversidad (Poner et al., 2001) y para afrontar desafíos y 
retos de la sociedad actual como la crisis de la educación, biodiversidad y de los programas públicos (conciencia ambiental) (Krishtalka \& Humphrey, 2000).

El Museo de Zoología de la Universidad de Costa Rica (MZUCR) se funda en 1966 (García, 2009) y alberga colecciones que son patrimonio científico, cultural e histórico de la Universidad de Costa Rica y del Estado Costarricense (PEMZ, 2016). Las colecciones presentes en este museo comprenden a la gran mayoría de los grupos taxonómicos de animales invertebrados y vertebrados de Costa Rica (PEMZ, 2016). El MZUCR actualmente cuenta con 24 colecciones que contienen más de cinco millones de especímenes, y más de 13000 especies identificadas. Las primeras colecciones datan de 1960 e incluyen peces, reptiles, anfibios, poliquetos, crustáceos y equinodermos (PEMZ, 2016). Además, de acuerdo a la Ley de Conservación de la vida silvestre de Costa Rica (artículo 46, Ley No. 4594 del 22 de julio de 1970), el MZUCR en conjunto con el Museo Nacional son los repositorios oficiales de cualquier espécimen biológico que haya sido obtenido con fines de recolecta científica o cultural.

Los equinodermos (estrellas pluma, estrellas de mar, estrellas quebradizas, erizos de mar y pepinos de mar) son animales deuterostomados exclusivamente marinos. El filo Echinodermata, se caracteriza por poseer una piel cubierta de espinas, un endoesqueleto calcáreo, una simetría pentaradial en los adultos y un sistema vascular acuífero único en el reino animal. El filo está compuesto por cinco clases: Crinoidea, Asteroidea, Ophiuroidea, Echinoidea, y Holothuroidea. Posee aproximadamente 7000 especies vivientes y unas 13000 fósiles en varias clases ya extintas (Pawson, 2007). Hasta el presente trabajo, para Costa Rica se han reportado 229 especies de equinodermos, 187 del Pacífico (127 de la Isla del Coco y 106 del Pacífico continental) y 44 del Caribe (Alvarado \& Cortés, 2009; Alvarado, Barraza, \& Sancho-Mejías, 2013).

Se han realizado varias revisiones de la diversidad de equinodermos de Costa Rica
(Alvarado, Solis-Marin, \& Ahearn, 2008, 2010; Alvarado \& Cortés, 2009; Alvarado et al., 2013), y a pesar de que estos estudios se basan en información de colecciones biológicas de museos, ninguna hace un análisis del aporte de cada una de ellas al conocimiento de la diversidad que se posee. Por lo tanto, el objetivo del presente trabajo es realizar un análisis de la contribución de varios museos de historia natural a nivel mundial al conocimiento de los equinodermos de Costa Rica y evaluar el aporte del Museo de Zoología de la Universidad de Costa Rica (MZUCR) a dicho conocimiento. Así mismo, identificar en la colección de equinodermos del MZUCR los distritos costeros más diversos, así como las zonas donde existen vacíos de conocimiento.

\section{MATERIALES Y MÉTODOS}

Para construir el listado taxonómico actualizado de equinodermos de Costa Rica se visitaron las siguientes bases de datos de colecciones biológicas: 1) Academia de las Ciencias de California (CAS) (http://researcharchive. calacademy.org/research/izg/iz_coll_db/index. asp); 2) Instituto Oceanográfico Scripps (SIO) (https://scripps.ucsd.edu/collections/benthicinvertebrate); 3) Museo Nacional de Historia Natural del Instituto Smithsoniano (NHMH) (http://collections.nmnh.si.edu/); 4) Museo de Zoología Comparada de Harvard (MZC); 5) Colección Nacional de equinodermos "Dra. Ma. Elena Caso" de la Universidad Nacional Autónoma de México (ICML-UNAM); y 6) Museo de Zoología de la Universidad de Costa Rica (MZUCR). Las bases de datos del MZC, ICML-UNAM y MZUCR fueron facilitadas por los curadores respectivos de las colecciones. Es posible que haya material de Costa Rica en el Museo de Historia Natural de Dinamarca (NCD) y en el Museo de Historia Natural de los Ángeles (LACM), sin embargo, no hubo acceso a dichas colecciones en línea ni a través de los curadores responsables. Así mismo, se revisó una serie de publicaciones que hacen referencia a registros de especies para las costas de Costa Rica y que no aparecen depositados 
en ninguna colección (Verrill, 1867; Lütken \& Mortensen, 1899; A. Agassiz, 1904; Ludwig, 1905; Fisher, 1928; Nielsen, 1932; H. L, Clark, 1940, 1948; Ziesenhenne, 1940; Deichmann, 1958; Hertlein, 1963; Bakus, 1974; Wellington, 1974; Maluf, 1988, 1991; Hendler, 1995; Lessios, Kessing \& Robertson, 1999; Cortés \& León, 2002; Alvarado \& Cortés, 2009). Esta literatura aparece enumerada en la sección de Referencias y se indica en el listado taxonómico del Cuadro 2.

Se analizó el esfuerzo de muestreo del MZUCR por medio de gráficas en las que se representaron las coordenadas de colecta de los diferentes especímenes depositados en esta colección. El análisis se realizó a través de la menor categoría administrativa territorial de Costa Rica, graficando el número de especies colectadas por distrito costero.

\section{RESULTADOS Y DISCUSIÓN}

Con base en la revisión de colecciones biológicas de museos, se actualizó el listado taxonómico de equinodermos para Costa Rica para un total de 293 especies, 152 géneros, 75 familias, 30 órdenes y cinco clases (Cuadro 1). La costa Pacífica de Costa Rica posee 146 especies, seguida por la Isla del Coco con 134 y la costa Caribe con 65 (Cuadro 1). Se determinaron 55 especies en común entre el Pacífico continental y la Isla del Coco, mientras que entre el Pacífico y el Caribe solo se observó una especie en común (Ophiactis savignyi) (Fig. 1). Previamente se había informado de un total de 229 especies de equinodermos, 187 del Pacífico y 44 del Caribe (Alvarado \& Cortés, 2009; Alvarado, Barraza, \& Sancho-Mejías, 2013). Hay un incremento de 39 especies para el Pacífico, 21 para el Caribe y ocho para la Isla del Coco (Cuadro 1, 2). Este aumento en el número especies se debe al aumento en esfuerzo de muestreo, pero a su vez a una revisión más exhaustiva de los ejemplares de la colección del MZUCR por parte de curadores visitantes.

Holothuria resultó ser el género más diverso con 25 especies. Para la Clase Crinoidea el mayor número de especies reportadas se da en la costa Caribe (Fig. 1). Siguiendo este criterio, los asteroideos se encuentran mejor representados en la isla del Coco. Mientras que las Clases Ophiuroidea, Echinoidea y Holothuroidea presentaron una mayor riqueza en la costa Pacífica.

El MZUCR tiene el $54.9 \%$ del total de especies de equinodermos que han sido colectados en Costa Rica. El resto de especies están repartidas en las siguientes instituciones: el Museo Nacional de Historia Natural del Instituto Smithsoniano (NMNH) (35.1\%), el Museo de Zoología Comparada de Harvard (MZC) (19.2\%), la Colección Nacional de equinodermos "Dra. Ma. Elena Caso" de la Universidad Nacional Autónoma de México (ICML-UNAM) (12.7\%), el Instituto Oceanográfico Scripps (SIO) (5.2\%), y en la Academia

CUADRO 1

Especies, géneros, familias y órdenes por clase de equinodermos para Costa Rica

TABLE 1

Species, genera, families and orders by class of echinoderms for Costa Rica

\begin{tabular}{|c|c|c|c|c|c|c|}
\hline & Crinoidea & Asteroidea & Ophiuroidea & Echinoidea & Holothuroidea & Total \\
\hline Orden & 2 & 8 & 2 & 12 & 6 & 30 \\
\hline Familia & 5 & 22 & 12 & 19 & 17 & 75 \\
\hline Género & 7 & 40 & 36 & 34 & 36 & 152 \\
\hline Especie & 9 & 56 & 90 & 65 & 73 & 293 \\
\hline Caribe & 5 & 8 & 25 & 14 & 13 & 65 \\
\hline Isla del Coco & 1 & 31 & 33 & 31 & 38 & 134 \\
\hline Pacífico & 4 & 28 & 49 & 32 & 40 & 153 \\
\hline
\end{tabular}




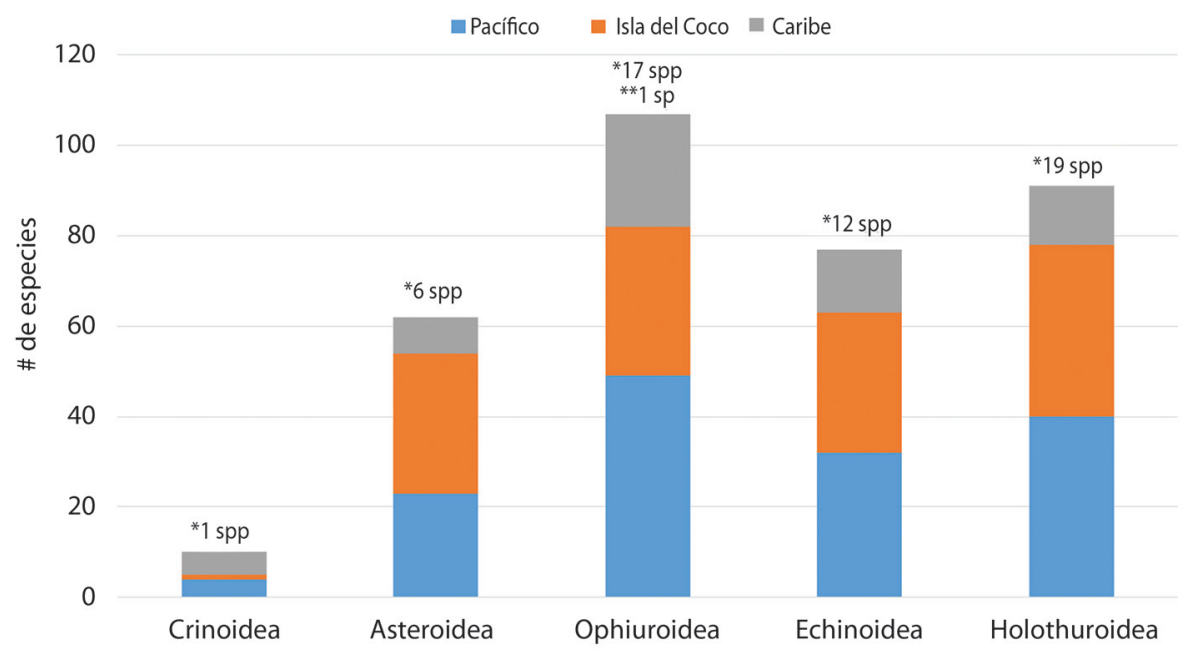

Fig. 1. Número de especies de equinodermos registradas en el Caribe, Pacífico e Isla del Coco por clase. (*) especies en común entre el Pacífico e Isla del Coco; $(* *)$ especies en común entre costa Pacífica y Caribe.

Fig. 1. Number of species of echinoderms recorded in the Caribbean, Pacific and Cocos Island by class. $(*)$ Species in common between the Pacific and Cocos Island; $(* *)$ species in common between the Pacific coast and the Caribbean coast.

\section{CUADRO 2}

Listado taxonómico de los equinodermos de Costa Rica. CAS: Academia de la Ciencias de California (CAS); SIO: Instituto Oceanográfico Scripps; ICML-UNAM: Colección Nacional de equinodermos "Dra. Ma. Elena Caso" de la Universidad Nacional Autónoma de México; MZC: Museo de Zoología Comparada de Harvard; NHMH: Museo Nacional de Historia Natural del Instituto Smithsoniano; MZUCR: Museo de Zoología de la Universidad de Costa Rica; PAC: Pacífico; CAR: Caribe; IC: Isla del Coco. LIT*: Literatura. (*) número de la referencia

TABLE 2

Taxonomic list of Costa Rican echinoderms. CAS: California Academy of Sciences (CAS); SIO: Scripps Oceanographic Institute; ICML-UNAM: National Collection of Echinoderms "Dra. Ma. Elena Caso" of the National Autonomous

University of Mexico; MZC: Harvard Museum of Comparative Zoology; NHMH: National Museum of Natural History, Smithsonian Institute; MZUCR: Zoology Museum of the University of Costa Rica; PAC: Pacific; CAR: Caribbean; IC: Isla del Coco. LIT *: Literature. $(*)$ number of the reference

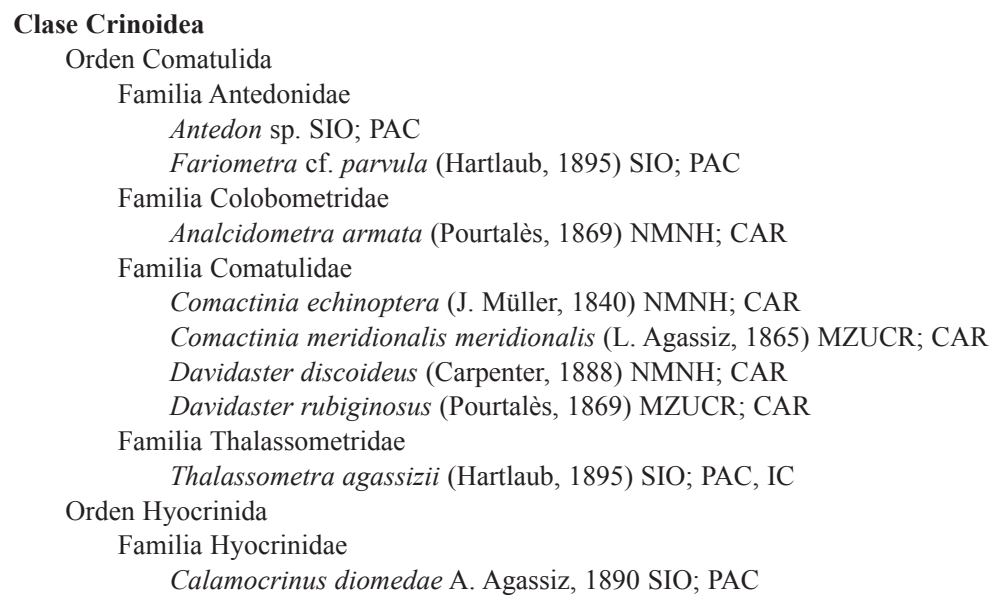




\section{Clase Asteroidea}

Orden Paxillosida

Familia Astropectinidae

Astropecten armatus Gray, 1840 ICML-UNAM, MZUCR, MZC; PAC

Astropecten articulatus (Say, 1825) LIT $^{18}$; CAR

Astropecten benthophilus Ludwig, 1905 NMHN; IC

Astropecten cingulatus Sladen, 1883 NMHN; CAR

Astropecten marginatus Gray, 1840 NMHN; CAR

Astropecten regalis Gray, 1840 ICML-UNAM, MZUCR; PAC

Astropecten sulcatus Ludwig, 1905 NMHN, MZC; IC

Leptychaster inermis (Ludwig, 1905) LIT ${ }^{8,12}$; IC

Thrissacanthias sp. MZUCR; PAC

Familia Luidiidae

Luidia armata Ludwig, 1905 NMNH, MZC; IC

Luidia columbia (Gray, 1840) MZUCR, MZC; PAC

Luidia latiradiata (Gray, 1871) MZUCR; PAC

Luidia tessellata Lütken, 1859 NMNH, MZUCR; PAC

Luidia senegalensis (Lamark, 1816) MZUCR, CAR

Luidia superba A.H. Clark, 1917 MZUCR; PAC

Familia Porcellanasteridae

Ermicaster pacificus (Ludwig, 1905) NMHN; IC

Porcellanaster ceruleus Wyville Thomson, 1877 NMHN; IC

Familia Pseudarchasteridae

Pseudarchaster sp. MZUCR; PAC

Orden Notomyotida

Familia Benthopectinidae

Benthopecten spinuliger (Ludwig, 1905) NMHN, MZC; IC

Pectinaster agassizi Ludwig, 1905 NMHN; IC

Orden Valvatida

Familia Acanthasteridae Acanthaster planci (Linnaeus, 1758) NMNH, MZUCR; IC

Familia Asterinidae Asterinides folium (Lütken, 1859) MZUCR; PAC

Familia Asterodiscididae Paulia horrida Gray, 1840 NMNH, MZC; IC

Familia Asteropsidae

Asteropsis carinifera (Lamarck, 1816) NMNH, MZUCR; PAC, IC

Poraniella echinulata (Perrier, 1881) NMNH; CAR

Familia Goniasteridae

Bathyceramaster elegans (Ludwig, 1905) NMNH; IC

Nymphaster diomedeae Ludwig, 1905 NMNH; IC

Nymphaster sp. SIO; PAC

Pillsburiaster ernesti (Ludwig, 1905) NMNH; IC

Familia Mithrodiidae

Mithrodia bradleyi Verrill, 1870 MZUCR; IC

Familia Ophidiasteridae

Leiaster teres (Verrill, 1871) MZUCR; IC

Linckia columbiae Gray, 1840 MZUCR; PAC, IC

Linckia guildingi Gray, 1840 NMNH, MZUCR; CAR

Linckia laevigata (Linnaeus, 1758) CAS; IC

Linckia multifora (Lamarck, 1816) CAS; IC

Narcissia sp. ICLM-UNAM, MZUCR, MZC; PAC

Pharia pyramidata (Gray, 1840) CAS, ICLM-UNAM, MZUCR, MZC; PAC, IC

Phataria unifascialis (Gray, 1840) ICLM-UNAM, MZUCR, MZC; PAC

Tamaria obstipa Ziesenhenne, 1942 LIT $^{10,15,19}$; PAC, IC 
Familia Oreasteridae

Nidorellia armata (Gray, 1840) MZUCR; PAC, IC

Oreaster reticulatus (Linnaeus, 1758) LIT ${ }^{6,18}$; CAR

Pentaceraster cumingi (Gray, 1840) MZUCR; PAC, IC

Familia Pterasteridae

Pteraster diaphanus (Ludwig, 1905) NMNH; IC

Orden Velatida

Familia Caymanostellidae

Caymanostella sp. SIO; PAC

Belyaevostella sp. SIO; PAC

Orden Spinulosida

Familia Echinasteridae

Echinaster (Othilia) aculeata (Gray, 1840) LIT ${ }^{17}$; PAC

Familia Pterasteridae

Hymenaster quadrispinosus Fisher, 1905 LIT $^{14}$; IC

Orden Forcipulatida

Familia Asteriidae

Sclerasterias heteropaes Fisher, 1924 NMHN; IC

Tarsaster cocosanus (Ludwig, 1905) NMHN, IC

Familia Heliasteridae

Heliaster solaris A.H. Clark, 1920 LIT $^{14,15}$; PAC

Familia Pedicellasteridae

Hydrasterias improvisus (Ludwig, 1905) NMHN; IC

Sclerasterias alexandri (Ludwig, 1905) NMHN; IC

Familia Zoroasteridae

Doraster constellatus Downey, 1970 NMHN; CAR

Familia Asteriidae

Coronaster marchenus Ziesenhenne, 1942 MZUCR, PAC

Orden Brisingida

Familia Brisingidae

Astrolirus panamensis (Ludwig, 1905) NMHN; IC

Orden Peripodida

Familia Xyloplacidae

Xyloplax sp. SIO; PAC

\section{Clase Ophiuroidea}

Orden Euryalida

Familia Gorgonocephalidae

Astrocaneum spinosum (Lyman, 1875) MZUCR; PAC

Astrophyton muricatum (Lamarck, 1816) MZUCR; CAR

Gorgonocephalus sp. SIO; PAC

Orden Ophiurida

Familia Ophiomyxidae

Ophiomyxa flaccida (Say, 1825) MZUCR; CAR

Ophiomyxa panamensis Lütken \& Mortensen, 1899 NMNH; IC

Familia Amphiuridae

Amphiodia oerstedi (Lütken, 1856) NMNH; PAC

Amphiodia tabogae Nielsen, 1932 NMNH; PAC, IC

Amphiodia vicina H.L. Clark, 1940 MZC; PAC

Amphiodia violacea (Lütken, 1856) NMNH; PAC, IC

Amphiodia (Amphispina) urtica (Lyman, 1860) NMNH, ICML-UNAM; PAC

Amphipholis pugetana (Lyman, 1860) ICML-UNAM, MZUCR; PAC

Amphipholis squamata (Delle Chiaje, 1828) NMNH; PAC

Amphiura arcystata H.L. Clark, 1911 LIT $^{15}$; IC

Amphiura (Amphiura) diomedeae Lütken \& Mortensen, 1899 ICML-UNAM, MZUCR; PAC

Microphiopholis geminata (Le Conte, 1851) MZUCR; PAC 
Microphiopholis platydisca (Nielsen, 1932) NMNH, MZC; PAC

Microphiopholis puntarenae (Lütken, 1856) LIT ${ }^{16,17}$; PAC

Ophiocnida hispida (Le Conte, 1851) MZUCR; PAC

Ophiophragmus marginatus (Lütken, 1856) ICML-UNAM; PAC

Ophiophragmus ophiactoides Ziesenhenne, 1940 ICML-UNAM; PAC

Ophiophragmus paucispinus Nielsen, 1932 LIT $^{15}$; IC

Ophiophragmus tabogensis Nielsen, 1932 NMNH, MZUCR; PAC

Ophiophragmus spp. NMNH; PAC, IC

Ophiostigma isocanthum (Say, 1825) LIT'; CAR

Ophiphragmus spp. MZUCR; PAC

Triplodia abdita (A.M. Clark, 1970) LIT ${ }^{14}$; IC

Familia Hemieuryalidae

Sigsbeia lineata Lütken \& Mortensen, 1899 ICML-UNAM, MZUCR, MZC; IC

Familia Ophiacanthidae

Ophiacantha phragma Ziesenhenne, 1940 LIT $^{10}$; IC

Ophiophthalmus normani (Lyman, 1879) ICML-UNAM; PAC

Ophiotoma paucispina (Lütken \& Mortensen, 1899) LIT ${ }^{13}$; IC

Ophiochondrus sp. SIO; PAC

Ophiomitra sp. SIO; PAC

Familia Ophiactidae

Hemipholis gracilis Verrill, $1867 \mathrm{NMNH}$; PAC

Ophiactis quinqueradia Ljungman, 1872 MZUCR; CAR

Ophiactis savignyi (J. Müller \& Troschel, 1842) NMNH, ICML-UNAM, MZUCR; PAC, IC, CAR

Ophiactis simplex (Le Conte, 1851) NMNH, MZUCR; PAC, IC

Familia Ophiocomidae

Ophiocoma aethiops Lütken, 1859 NMNH, ICML-UNAM, MZUCR, MZC; PAC, IC

Ophiocoma alexandri Lyman, 1860 NMNH, ICML-UNAM, MZUCR; PAC, IC

Ophiocoma echinata (Lamarck, 1816) MZUCR; CAR

Ophiocoma paucigranulata Devaney, 1974 MZUCR; CAR

Ophiocoma pumila Lütken, 1856 MZUCR; CAR

Ophiocoma wendtii J. Müller \& Troschel, 1842 MZUCR; CAR

Ophiocomella ophiactoides (H.L. Clark, 1901) MZUCR; CAR

Ophiocomella schmitti A.H. Clark, 1939 MZUCR; PAC, IC

Ophiocomella sexradia (Duncan, 1887) NMNH, MZUCR; PAC, IC

Ophiocomella sp. NMNH; CAR

Ophiopsila riisei Lütken, 1859 MZUCR; CAR

Ophiopsila hartmeyeri Koehler, 1913 ICML-UNAM; CAR

Familia Ophiodermatidae

Diopederma daniana (Verrill, 1867) NMNH, MZUCR, MZC; PAC, IC

Ophioderma appressa (Say, 1825) MZUCR; CAR

Ophioderma brevicauda Lütken, 1856 MZUCR; CAR

Ophioderma brevispina (Say, 1825) MZUCR; CAR

Ophioderma cinerea J. Müller \& Troschel, 1842 MZUCR; CAR

Ophioderma panamensis Lütken, 1859 ICML-UNAM, MZUCR, MZC; PAC, IC

Ophioderma rubicunda Lütken, 1856 MZUCR; CAR

Ophioderma sodipallaresi Caso, 1986 MZUCR; PAC

Ophioderma teres (Lyman, 1860) NMNH, ICML-UNAM, MZUCR, MZC; PAC

Ophioderma variegata Lütken, 1856 ICML-UNAM, MZUCR, MZC; PAC, IC

Ophiopaepale diplax (Nielsen, 1932) NMNH, ICML-UNAM, MZUCR, MZC; PAC

Familia Ophionereididae

Ophionereis albomaculata E. A. Smith, 1877 ICML-UNAM; PAC, IC

Ophionereis annulata (Le Conte, 1851) NMNH, ICML, MZUCR, MZC; PAC, IC

Ophionereis eurybrachiplax H.L. Clark, 1911 LIT $^{14}$; IC

Ophionereis perplexa Ziesenhenne, 1940 MZUCR; PAC

Ophionereis reticulata (Say, 1825) MZUCR; CAR 
Familia Ophiolepididae

Ophiolepis grisea H.L. Clark, 1940 MZC; PAC

Ophiolepis impressa Lütken, 1859 MZUCR; CAR

Ophiolepis pacifica Lütken, 1856 MZUCR; PAC, IC

Ophiolepis plateia Ziesenhenne, 1940 MZC; PAC

Ophiolepis variegata Lütken, 1856 NMNH, MZUCR, MZC; PAC

Ophiomusium glabrum Lütken \& Mortensen, 1899 NMNH, MZC; IC

Ophiomusium lymani Wyville-Thomson, 1873 NMNH, MZC; IC

Ophiozonella alba (Lütken \& Mortensen, 1899) NMNH; IC

Familia Ophiothrichidae

Ophiothela gracilis Nielsen, 1932 MZC; PAC

Ophiothela mirabilis Verrill, 1867 NMNH, ICML-UNAM, MZUCR; PAC

Ophiothela sp. MZUCR; IC

Ophiothrix cimar Hendler, 2005 NMNH, MZUCR; CAR

Ophiothrix galapagensis Lütken \& Mortensen, 1899 MZUCR; PAC

Ophiothrix lineata Lyman, 1860 MZUCR; CAR

Ophiothrix stri Hendler, 2005 MZUCR; CAR

Ophiothrix (Acanthophiothrix) suensoni Lütken, 1856 MZUCR; CAR

Ophiothrix (Ophiothrix) angulata (Say, 1825) MZUCR; CAR

Ophiothrix (Ophiothrix) oerstedii Lütken, 1856 MZUCR; CAR

Ophiothrix (Ophiothrix) rudis Lyman, 1874 MZUCR; PAC

Ophiothrix (Ophiothrix)spiculata Le Conte, 1851 CAS, NMNH, MZUCR, MZC; PAC, IC

Familia Ophiuridae

Amphiophiura abcisa (Lütken \& Mortensen, 1899) NMNH, MZC; IC

Ophiocten hastatum Lyman, 1878 NMNH; IC

Ophiura sp. SIO, NMNH; PAC, IC

Ophiura (Ophiuroglypha) irrorata irrorata (Lyman, 1878) NMNH; IC

Ophiura (Ophiura) nana (Lütken \& Mortensen, 1899) NMNH; IC

Ophiambix sp. SIO; PAC

\section{Clase Echinoidea}

Orden Cidaroida

Familia Cidaridae

Eucidaris galapagensis Döderlein, 1887 LIT $^{11}$; IC

Eucidaris thouarsii (L. Agassiz \& Desor, 1846) CAS, NMNH, MZUCR; PAC, IC

Eucidaris tribuloides (Lamarck, 1816) NMNH, MZUCR; CAR

Centrocidaris doederleini (A. Agassiz, 1898) NMNH, MZUCR; IC

Hesperocidaris asteriscus H.L. Clark, 1948 MZUCR; PAC, IC

Hesperocidaris dubia (H.L. Clark, 1907) MZC; PAC, IC

Hesperocidaris houstoniana A.H. Clark, 1939 NMNH; IC

Hesperocidaris panamensis (A. Agassiz, 1898) NMNH, ICML-UNAM, MZUCR, MZC; PAC, IC

Hesperocidaris perplexa (H.L. Clark, 1907) MZUCR; PAC

Orden Aspidodiadematoida

Familia Aspidodiadematidae

Plesiodiadema horridum (A. Agassiz, 1898) NMNH, MZC; IC

Orden Diadematoida

Familia Diadematidae

Astropyga pulvinata (Lamarck, 1816) NMNH, MZUCR; PAC, IC

Centrostephanus sp. NMNH, IC

Diadema antillarum (Philippi, 1845) MZUCR; CAR

Diadema mexicanum A. Agassiz, 1863 NMNH, MZUCR; PAC, IC

Echinothrix calamaris (Pallas, 1774) NMNH; IC

Echinothrix diadema (Linnaeus, 1758) NMNH, MZUCR; IC

Orden Salenioida

Familia Saleniidae

Salenocidaris miliaris (A. Agassiz, 1898) LIT $^{15}$; IC 
Orden Arbacioida

Familia Arbaciidae Arbacia stellata (Blainville, 1825; ?Gmelin, 1791) MZUCR: PAC

Orden Camarodonta

Familia Echinometridae

Echinometra lucunter (Linnaeus, 1758) MZUCR; CAR

Echinometra mathaei oblonga (Blainville, 1825) MZUCR; IC

Echinometra vanbrunti A. Agassiz, 1863 CAS, MZUCR, MZC; PAC, IC

Echinometra viridis A. Agassiz, 1863 MZUCR; CAR

Familia Toxopneustidae

Lytechinus pictus (Verrill, 1867) LIT ${ }^{4}$; IC

Lytechinus variegatus (Lamarck, 1816) ICML-UNAM, MZUCR; CAR

Toxopneustes roseus (A. Agassiz, 1863) MZUCR ; PAC, IC

Tripneustes depressus A. Agassiz, 1863 NMNH, MZUCR ; PAC, IC

Tripneustes ventricosus (Lamarck, 1816) MZUCR; CAR

Orden Echinoneoida

Familia Echinoneidae

Echinoneus cyclostomus Leske, 1778 MZUCR; CAR

Orden Echinothurioida

Familia Echinothuriidae

Tromikosoma hispidum (A. Agassiz, 1898) NMNH; IC

Orden Clypeasteroida

Familia Clypeasteridae

Clypeaster euclastus H.L. Clark, 1941 NMNH; CAR

Clypeaster europacificus H.L. Clark, 1914 MZUCR; MZC; PAC

Clypeaster ochrus H.L. Clark, 1914 MZUCR; IC

Clypeaster rotundus (A. Agassiz, 1863) CAS, MZUCR; PAC

Clypeaster speciosus Verrill, 1870 LIT $^{4}$; IC

Clypeaster subdepressus (Gray, 1825) NMNH, MZUCR; CAR

Familia Mellitidae

Encope laevis H.L. Clark, 1948 CAS, MZUCR; PAC

Encope michelini L. Agassiz, 1841 MZUCR; PAC

Encope micropora cocosi H.L. Clark, 1948 MZC; IC

Encope micropora irregularis H.L. Clark, 1948 LIT $^{5}$; PAC

Encope micropora micropora L. Agassiz, 1841 CAS, MZUCR, MZC; PAC, IC

Encope perspectiva L. Agassiz, 1841 MZUCR; PAC

Encope wetmorei A.H. Clark, 1946 CAS; PAC

Lanthonia grantii (Mortensen, 1948) MZUCR; PAC

Lanthonia longifissa (Michelin, 1858) CAS, ICML-UNAM; PAC

Leodia sexiesperforata (Leske, 1778) LIT $^{2}$; CAR

Mellita kanakoffi Durham, 1961 CAS; PAC

Mellita quinquiesperforata (Leske, 1778) CAS, NMNH, MZUCR; CAR

Mellitella stokesii (L. Agassiz, 1841) NMNH, MZUCR; PAC

Orden Cassiduloida

Familia Cassidulidae

Cassidulus pacifica (A. Agassiz, 1863) LIT $^{2}$; PAC

Orden Spatangoida

Familia Asterostomatidae

Argopatagus aculeata (A. Agassiz, 1898) LIT ${ }^{1,14}$; IC

Familia Brissidae

Brissopsis pacifica (A. Agassiz, 1898) MZUCR; PAC, IC

Brissopsis atlantica Mortensen, 1907 NMNH; CAR

Brissus latecarinatus (Leske, 1778) MZUCR; PAC

Brissus obesus Verrill, 1867 MZUCR; PAC

Brissus unicolor (Leske, 1778) MZUCR; CAR

Meoma ventricosa ventricosa (Lamarck, 1816) MZUCR; CAR 
Meoma ventricosa grandis Gray, 1851 LIT $^{5}$; PAC, IC

Plagiobrissus grandis (Gmelin, 1788) MZUCR; IC

Plagiobrissus pacificus H.L. Clark, 1940 MZUCR; IC

Familia Prenastridae

Agassizia scrobiculata Valenciennes, 1846 MZUCR; PAC

Familia Schizasteridae

Moira atropos clotho (Michelin, 1855) LIT ${ }^{4,5}$; PAC

Familia Loveniidae

Araeolampas hastata (A. Agassiz, 1898) MZC; IC

Lovenia cordiformis A. Agassiz, 1872 MZUCR; IC

Familia Macropneustidae

Phrissocystis aculeata A. Agassiz, 1898 NMNH, MZC; IC

Orden Pedinoida

Familia Pedinidae

Caenopedina sp. SIO; PAC

\section{Clase Holothuroidea}

Orden Dendrochirotida

Familia Cucumariidae

Abyssocucumis abyssorum (Théel, 1886) NMNH; IC

Cucumaria flamma Solís-Marín \& Laguarda-Figueras, 1999 MZUCR; PAC

Cucumaria sp. NMNH; PAC

Leptopentacta nina Deichmann, 1941 MZUCR; PAC

Leptopentacta deichmannae Domantay, 1953 (nomen dubium) NMNH; CAR

Neocucumis veleronis (Deichmann, 1941) MZUCR; PAC

Pseudocnus californicus (Semper, 1868) ICML-UNAM, MZUCR; PAC

Pseudocnus dubiosus (Semper, 1868) MZUCR; PAC

Trachythyone peruana (Semper, 1868) MZUCR; PAC

Familia Phyllophoridae

Pentamera beebei Deichmann, 1938 MZC; PAC

Pentamera chierchiae (Ludwig, 1887) MZUCR, MZC; PAC, IC

Pentamera zacae Deichmann, 1938 MZUCR; PAC

Familia Psolidae

Lissothuria caboblanquensis Arriaga-Ochoa, Alvarado, Solís-Marín, Laguarda-Figueras, 2014 MZUCR; PAC

Lissothuria ornata Verrill, 1867 MZUCR, MZC; PAC, IC

Psolus diomedeae Ludwig, 1893 NMNH, MZC; PAC, IC

Familia Sclerodactylidae

Afrocucumis ovulum (Selenka, 1867) MZUCR, MZC; PAC

Euthyonidiella zacae (Deichmann, 1938) MZUCR; PAC

Neothyone gibber (Selenka, 1867) ICML-UNAM, MZUCR; PAC

Neothyone gibbosa Deichmann, 1941 MZUCR, MZC; PAC

Neothyone panamensis (Ludwig, 1887) MZUCR; PAC, IC

Pachythyone lugubris (Deichmann, 1939) MZUCR; PAC

Orden Dactylochirotida

Familia Ypsilothuriidae

Ypsilothuria bitentaculata (Ludwig, 1893) SIO; PAC, IC

Orden Aspidochirotida

Familia Holothuriidae

Actinopyga agassizii (Selenka, 1867) MZUCR; CAR

Bohadschia? LIT $^{3}$; IC

Holothuria (Cystipus) inhabilis Selenka, 1867 MZC; IC

Holothuria (Cystipus) rigida (Selenka, 1867) NMNH, MZUCR; PAC

Holothuria (Halodeima) atra Jaeger, 1833 MNNH, MZUCR; PAC, IC

Holothuria (Halodeima) grisea Selenka, 1867 MZUCR; CAR

Holothuria (Halodeima) inornata Semper, 1868 MZUCR; PAC, IC

Holothuria (Halodeima) kefersteinii (Selenka, 1867) ICML-UNAM, MZUCR, MZC; PAC, IC 
Holothuria (Halodeima) mexicana Ludwig, 1875 MZUCR; CAR

Holothuria (Lessonothuria) pardalis Selenka, 1867 NMNH, ICML-UNAM, MZUCR, MZC; PAC, IC Holothuria (Mertensiothuria) hilla Lesson, 1830 ICML-UNAM, MZUCR, MZC; PAC, IC

Holothuria (Mertensiothuria) leucospilota (Brandt, 1835) NMNH, MZUCR, MZC; IC

Holothuria (Platyperona) difficilis Semper, 1868 NMNH, ICML-UNAM, MZC; PAC, IC

Holothuria (Platyperona) parvula (Selenka, 1867) MZUCR; CAR

Holothuria (Selenkothuria) erinacea Semper, 1868 MZC; IC

Holothuria (Selenkothuria) glaberrima Selenka, 1867 MZUCR; CAR

Holothuria (Selenkothuria) lubrica Selenka, 1867 NMNH, ICML-UNAM, MZUCR, MZC; PAC, IC

Holothuria (Selenkothuria) portovallartensis Caso, 1954 MZUCR; PAC

Holothuria (Selenkothuria) theeli Deichmann, 1938 LIT7; IC

Holothuria (Semperothuria) imitans Ludwig, 1875 ICML-UNAM, MZUCR; PAC, IC

Holothuria (Semperothuria) languens Selenka, 1867 MZUCR, MZC; PAC

Holothuria (Semperothuria) surinamensis Ludwig, 1875 MZUCR; CAR

Holothuria (Stauropora) pluricuriosa Deichmann, 1937 NMNH, MZUCR; PAC, IC

Holothuria (Theelothuria) paraprinceps Deichmann, 1937 LIT $^{7}$; IC

Holothuria (Thymiosycia) arenicola Semper, 1868 NMNH, MZUCR, MZC; PAC, IC

Holothuria (Thymiosycia) impatiens (Forskål, 1775) ICML-UNAM, MZUCR, MZC; PAC, IC

Holothuria (Vaneyothuria) zacae Deichmann, 1937 MZUCR, MZC; PAC, IC

Labidodemas americanum Deichmann, 1938 ICML-UNAM, MZUCR, MZC; PAC, IC

Labidodemas maccullochi (Deichmann, 1958) ICML-UNAM, MZUCR; PAC

Familia Stichopodidae

Isostichopus fuscus (Ludwig, 1875) MZUCR; PAC, IC

Stichopus horrens Selenka, 1867 MZUCR; IC

Familia Synallactidae

Bathyplotes sp. NMNH; CAR

Meseres macdonaldi Ludwig, 1893 (nomen dubium) NMNH; IC

Mesothuria multipes (Ludwig, 1893) NMNH; IC

Paroriza sp. NMNH; CAR

Pseudostichopus mollis Théel, 1886 NMNH, ICML-UNAM, MZC; IC

Pseudostichopus peripatus (Sluiter, 1901) NMNH; IC

Orden Elasipodida

Familia Deimatidae

Deima validum pacificum Ludwig, 1894 NMNH; IC

Oneirophanta setigera (Ludwig, 1893) NMNH; IC

Familia Elpidiidae

Peniagone vitrea Théel, 1882 NMNH; IC

Familia Laetmogonidae

Pannychia moseleyi Théel, 1882 NMNH; IC

Familia Pelagothuriidae

Pelagothuria natatrix Ludwig, 1893 LIT $^{15}$; IC

Familia Psychropotidae

Benthodytes sanguinolenta Théel, 1882 NMNH; IC

Orden Molpadiida

Familia Caudinidae

Paracaudina chilensis (Müller, 1850) MZC; PAC

Familia Molpadiidae

Molpadia musculus (Risso, 1826) NMNH; IC

Molpadia sp. NMNH; CAR

Orden Apodida

Familia Chiridotidae

Chiridota pacifica Heding, 1928 MZUCR; IC

Chiridota rotifera (Pourtalès, 1851) MZUCR; CAR

Familia Synaptidae

Synaptula hydriformis (Lesueur, 1824) MZUCR; CAR

Euapta lappa (J. Müller, 1850) MZUCR; CAR

Euapta godeffroyi (Semper, 1868) MZUCR; PAC 
de la Ciencias de California (CAS) (4.8\%). A su vez hay $9.6 \%$ de especies que no aparecen en ningún museo, pero están registradas en la literatura (Verrill, 1867; Lütken \& Mortensen, 1899; Agassiz, 1904; Ludwig, 1905; Fisher, 1928; Nielsen, 1932; Clark HL, 1940, 1948; Ziesenhenne, 1940; Deichmann, 1958; Hertlein, 1963; Bakus, 1974; Wellington, 1974; Maluf, 1988, 1991; Hendler, 1995; Lessios, Kessing \& Robertson, 1999; Cortés \& León, 2002; Alvarado \& Cortés, 2009), dentro de las cuales hay varias que pueden ser registros dudosos. Entre ellos está el caso del pepino de mar Bohadschia? que está reportado por Bakus (1974) para la Iisla del Coco en un estudio ecológico. Esta especie no está presente en ninguna colección y no está reportada para ninguna otra localidad del Pacífico Oriental Tropical (SolísMarín et al., 2013). Por otra parte, existen especies que la dificultad en su colecta, no se han podido ingresar a ninguna colección biológica, pero que si han sido observadas. Gracias al uso del submarino DeepSee en la Isla del Coco
(Cortés \& Blum, 2008), se ha tenido acceso a colectas e imágenes de muy alta definición de equinodermos de aguas profundas como es el caso del pepino de mar Pelagothuria natatrix (Alvarado et al., in prep.). Este tipo de tecnologías ha permitido confirmar la presencia de especies que han sido registradas únicamente en la literatura.

El MZUCR posee el mayor número de especies de las clases Ophiuroidea, Echinoidea y Holothuroidea (Fig. 2). Por su parte, el $\mathrm{NMNH}$ es que el alberga el mayor número de especies de asteroideos, mientras que el SIO es el más completo en crinoideos. La segunda colección más importante, luego del MZUCR, es la del NMNH (Fig. 2).

Sin lugar a dudas, el MZUCR resguarda la colección más importante de equinodermos de Costa Rica. Esta colección está conformada por un total de 157 especies, en 1173 lotes y 4316 ejemplares. El esfuerzo de colecta e investigación realizado durante los 50 años de existencia de este museo, indican que, en Costa

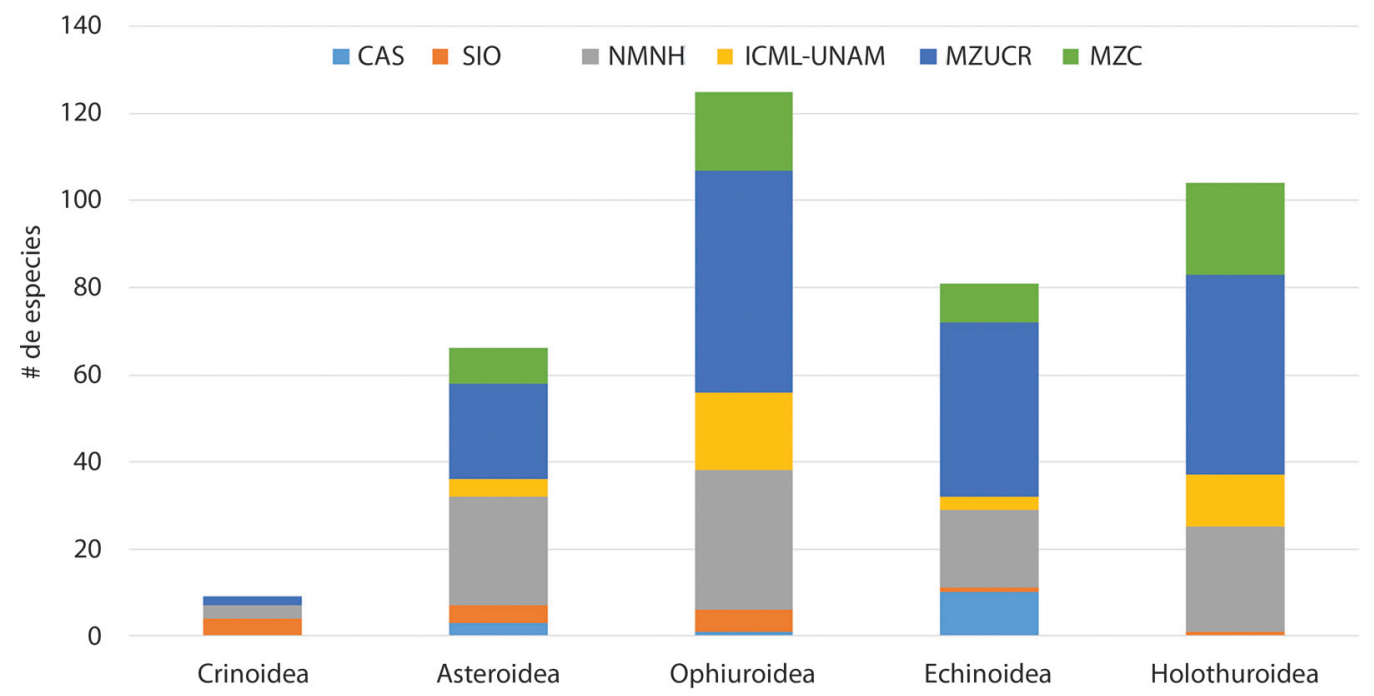

Fig. 2. Número de especies de equinodermos por clase registradas en cada museo. CAS: Academia de la Ciencias de California; SIO: Instituto Oceanográfico Scripps; ICML-UNAM: Colección Nacional de equinodermos "Dra. Ma. Elena Caso" Nacional Autónoma de México; MZC: Museo de Zoología Comparada de Harvard; NMNH: Museo Nacional de Historia Natural del Instituto Smithsoniano; MZUCR: Museo de Zoología de la Universidad de Costa Rica.

Fig. 2. Number of species of echinoderms per class recorded in each museum. CAS: California Academy of Sciences; SIO: Scripps Oceanographic Institute; ICML-UNAM: National Collection of Echinoderms "Dra. Ma. Elena Caso" of the National Autonomous University of Mexico; MZC: Harvard Museum of Comparative Zoology; NMNH: National Museum of Natural History, Smithsonian Institute; MZUCR: Zoology Museum of the University of Costa Rica. 


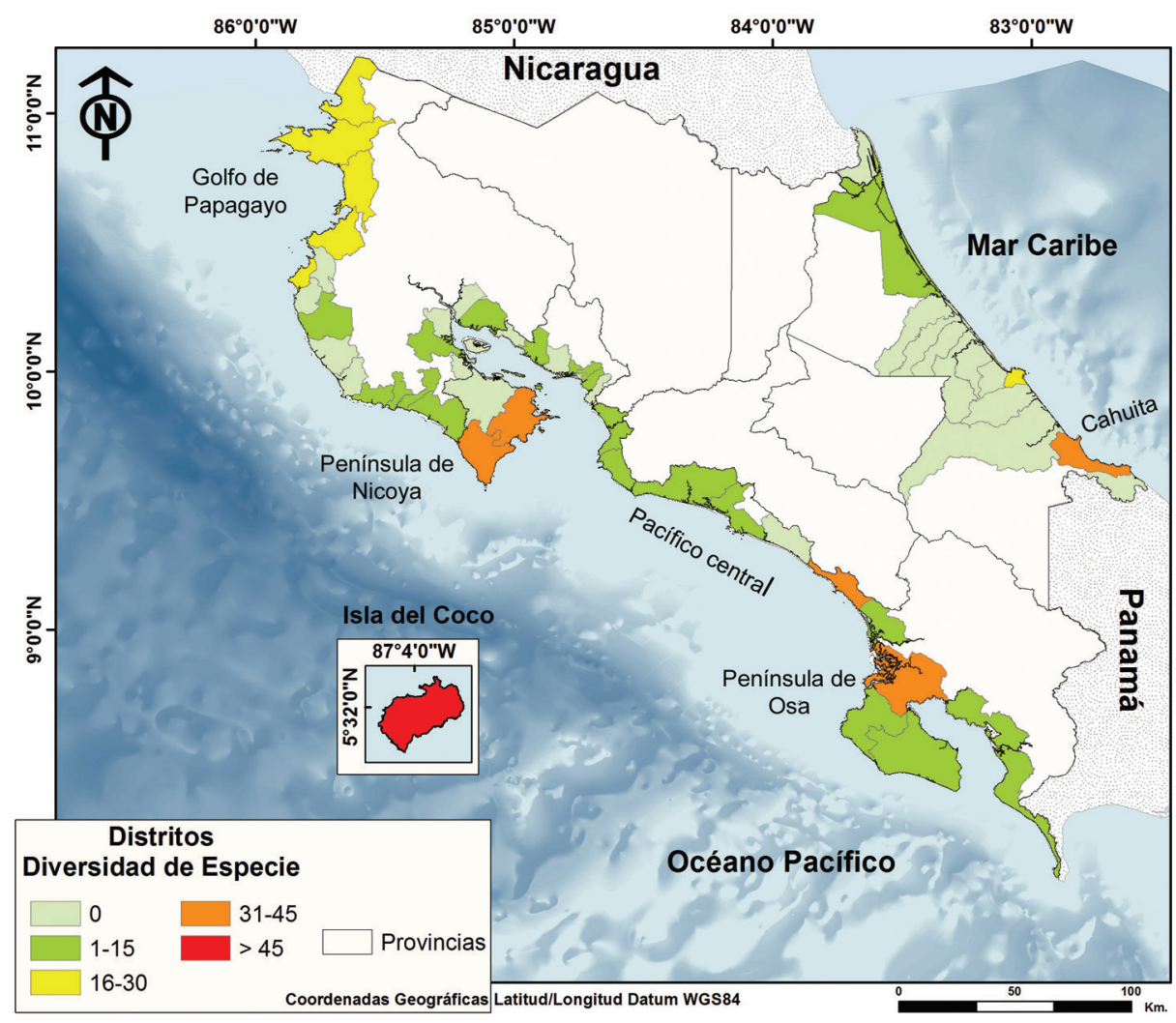

Fig. 3. Diversidad de especies de equinodermos por distrito costero de Costa Rica.

Fig. 3. Diversity of echinoderm species by coastal district of Costa Rica.

Rica para este grupo de organismos, las zonas más diversas se encuentran en la Isla del Coco, la Península de Osa y el Caribe Sur (Fig. 3). Estas zonas representan las regiones de mayor diversidad de ecosistemas en Costa Rica, y a su vez las más estudiadas y conservadas (Cortés \& Wehrtmann, 2009; Alvarado, Cortés, Esquivel, \& Salas, 2012; Cortés, 2016a, b, c). La figura 3 indica el esfuerzo de muestreo de 50 años de investigación, así como zonas poco estudiadas (Caribe Norte y Pacífico Central), convirtiéndolas en vacíos de conocimiento y puntos prioritarios de conservación (Alvarado, Herrera, Corrales, Asch, \& Paaby, 2011). Se debe aumentar el esfuerzo de muestreo en estas zonas, y a su vez en el mar profundo tanto en la vertiente Caribe como en la del Pacífico, así como la cordillera volcánica submarina del Coco.

\section{AGRADECIMIENTOS}

El presente trabajo no hubiera sido posible sin el aporte de varios estudiantes y asistentes que han colaborado con la colecta, identificación de equinodermos para el Museo de Zoología, en especial S. Mena, C. Salas, J.C Azofeifa, M. Villasevil-Almarcha, T. SanchoMejías, y los estudiantes del curso de Taxonomía y Ecología de Equinodermos. A su vez, la colaboración de R. Vargas, O. Breddy, J. Cortés y C. Fernández ha servido para incrementar el número de especímenes. 


\section{RESUMEN}

El Museo de Zoología de la Universidad de Costa Rica (MZUCR) se funda en 1966 y alberga la colección de organismos vertebrados e invertebrados más completa de Costa Rica. El MZUCR cuenta actualmente con 24 colecciones que contienen más de cinco millones de especímenes, y más de 13000 especies identificadas. Las primeras colecciones datan 1960 e incluyen peces, reptiles, anfibios, poliquetos, crustáceos y equinodermos. Para este último grupo, el MZUCR posee un total de 157 especies, en 1173 lotes y 4316 ejemplares. Estas 157 especies representan el $54 \%$ del total de especies de equinodermos que posee Costa Rica (293 especies). El resto de especies están repartidas en las siguientes instituciones: Academia de la Ciencias de California (CAS) (4.8\%), Instituto Oceanográfico Scripps (SIO) (5.2\%), en la Colección Nacional de equinodermos "Dra. Ma. Elena Caso" de la Universidad Nacional Autónoma de México (ICML-UNAM) (12.7\%), Museo de Zoología Comparada de Harvard (MZC) (19.2\%), y en el Museo Nacional de Historia Natural del Instituto Smithsoniano (USNM) (35.1\%). Es posible que haya material de Costa Rica en el Museo de Historia Natural de Dinamarca (NCD) y en el Museo de Historia Natural de los Ángeles (LACM), sin embargo, no hubo acceso a dichas colecciones. A su vez hay $9.6 \%$ de especies que no aparecen en ningún museo, pero están reportadas en la literatura. Con base en esta revisión de colecciones se actualizó el listado taxonómico de equinodermos para Costa Rica que consta de 293 especies, 152 géneros, 75 familias, 30 órdenes y cinco clases. La costa Pacífica de Costa Rica posee 153 especies, seguida por la isla del Coco con 134 y la costa Caribe con 65. Holothuria resultó ser el género más rico con 25 especies.

Palabras claves: colecciones zoológicas; esfuerzo de muestreo; puntos de alta diversidad; vacíos de investigación; nuevos reportes

\section{REFERENCIAS}

(1) Agassiz, A. (1904). Reports on an exploration off the west coast of Mexico, Central and south America, and off Galapagos Islands, in charge of Alexander Agassiz, by the U.S. Fish Commission Steamer "Albatross”, during 1891, Lieut. Commander Z.L. Tanner, U.S.Commanding. XXXII. The Panamic deep sea echini. Memoirs of the Museum of Comparative Zoology at Harvard College, 31, 1-246.

Alvarado, J.J., Solis-Marin, F.A., \& Ahearn, C. (2008). Equinodermos (Echinodermata) del Caribe Centroamericano. Revista de Biología Tropical, 56 (Supl. 3), 37-55.

(2) Alvarado, J.J., \& Cortés, J. (2009). Echinoderms. Chapter IV, Part 36, p. 421-433. In: Wehrtmann, I.S. \& Cortés, J. (eds.). Marine Biodiversity of Costa Rica,
Central America. Monographiae Biologicae, Vol 86. Berlin: Springer Verlag.

Alvarado, J.J., Solis-Marin, F.A. \& Ahearn, C. (2010). Echinoderms (Echinodermata) diversity in the Pacific coast of Central America. Marine Biodiversity, $40,45-56$.

Alvarado, J.J., Herrera, B., Corrales, L., Asch, J. \& P. Paaby. (2011). Identificación de las prioridades de conservación de la biodiversidad marina y costera en Costa Rica. Revista de Biología Tropical, 59(2), 829-842.

Alvarado, J.J., Cortés, J., Esquivel, M.F. \& Salas, E. (2012). Costa Rica's Marine Protected Areas: status and perspectives. Revista de Biología Tropical, 60 (1), 129-142.

Alvarado J.J., Barraza, E., \& Sancho-Mejías, T. (2013). Chapter 3. Central America Echinoderms: diversity, ecology and future perspectives, p. 67-106. In: Alvarado, J.J. \& Solís-Marín, F.A. (ed.). Echinoderm Research and Diversity in Latin America. Berlin: Springer.

(3) Bakus, G.J. (1974). Toxicity in holothurians: a geographical pattern. Biotropica, 6, 229-236.

(4) Clark, H.L. (1940) Eastern Pacific Expeditions of the New York Zoological Society. XXI Notes on Echinoderms from the west coast of Central America. Zoologica, 25, 331-352.

(5) Clark, H.L. (1948). A report on the Echini of the warmer eastern Pacific, based on the collections of the Velero III. Allan Hancock Pacific Expedition, 8(5), 225-352.

Cortés, J. (2016a). Chapter 5. The Pacific Coastal and Marine Ecosystems, p.p. 97-138. In: Kappelle, M. (ed.) Costa Rican Ecosystems. Chicago: The University of Chicago Press.

Cortés, J. (2016b). Chapter 7. Isla del Coco: Coastal and Marine Ecosystems, p. 162-191. In: Kappelle, M. (ed.) Costa Rican Ecosystems. Chicago: The University of Chicago Press.

Cortés, J. (2016c). Chapter 17: The Caribbean Coastal and Marine Ecosystems, p. 591-619. In: Kappelle, M. (ed.) Costa Rican Ecosystems. Chicago: The University of Chicago Press.

(6) Cortés. J, \& León, A. (2002). Arrecifes coralinos del Caribe de Costa Rica. Santo Domingo de Heredia: INBio Editorial.

Cortés, J. \& Blum, S. (2008). Life to $450 \mathrm{~m}$ depth at Isla del Coco, Costa Rica. Revista de Biología Tropical, 56 (Suppl. 2), 189-206.

Cortés, J. \& Wehrtmann, I.S. (2009). Diversity of marine habitats of the Caribbean and Pacific of Costa Rica, 
p. 1-45. Wehrtmann, I.S. \& Cortés, J. (eds.). Marine Biodiversity of Costa Rica, Central America. Monographiae Biologicae, Vol 86. Berlin: Springer Verlag.

(7) Deichmann, E. (1958). The Holothuroidea collected by the Velero III and IV during the years 1932 to 1954. Part II Aspidochirota. Allan Hancock Pacific Expedition, 11(2), 253-349.

(8) Fisher, W.K. (1928). Sea stars from the Arcturus oceanographic expedition. Zoologica, 8, 487-493.

García, J.E. (2009). Breve historia de la Escuela de Biología de la Universidad de Costa Rica. Revista de Biología Tropical, 57 (supl. 1), 1-14.

(9) Hendler, G. (1995). New species of brittle stars from the Western Atlantic, Ophionereis vittata, Amphioplus sepultus, and Ophiostigma siva, and the designation of a neotype for Ophiostigma isocanthum (Say) (Echinodermata: Ophiuroidea). Natutal History Museum of Los Angeles County Contributions in Science, 458,1-19.

(10) Hertlein, L.G. (1963) Contribution to the biogeography of Cocos Island, including a bibliography. Proceeding of the California Academy of Science, 32, 123-235.

Krishtalka, L., \& Humphrey, P.S. (2000). Can natural history museums capture the future? BioScience, 50, 611-617.

Lane, M.A. (1996). Roles of natural history collections. Annals of the Missouri Botanical Garden, $83,536-545$.

(11) Lessios, H.A., Kessing, B.D., \& Robertson, D.R. (1999). Phylogeography of the pantropical sea urchin Eucidaris in relation to land barriers and ocean currents. Evolution, 53, 806-817.

Lister, A.M., \& Climate Change Research Group. (2011). Natural history collections as sources of longterm datasets. Trends in Ecology and Evolution, 26, 153-154.

(12) Ludwig, H. (1905). Asteroidea in: Explorations of "Albatross" in Tropical Pacific, 1891 and 1899-1900. Memoirs of the Museum of Comparative Zoology, 22(7), 1-290.

(13) Lütken, C.F. \& Mortensen, T. (1899). Reports on an exploration off the west coast of Mexico,Central America and South America, and off the Galápagos Islands, in charge of A. Agassiz, during 1891 on the "Albatross." XXV. The Ophiuridae. Memoirs of the Museum of Comparative Zoology, 23(2), 93-208.

(14) Maluf, L.Y. (1988). Composition and distribution of the central eastern Pacific echinoderms. Natural
History Museum of Los Angeles County, Technical Report, 2,1-242.

(15) Maluf, L.Y. (1991) Echinoderm fauna of the Galapagos Islands, p. 345-367. In: James, M.J. (ed.). Galapagos Marine Invertebrates: Taxonomy, Biogeography and Evolution in Darwin's Islands. New York: Plenum.

(16) Nielsen, E. (1932). Papers from Dr. Th. Mortensen's Pacific Expedition 1914-16. LIX. Ophiurans from the Gulf of Panama, California and the Strait of Georgia. Vidensk Medd Dansk naturh Forem Bd, 91, 240-346.

Pawson, D.L. (2007). Phylum Echinodermata. Zootaxa, $1668,749-764$

PEMZ. (2016). Plan Estratégico Museo de Zoología 20162021, Escuela de Biología. San José: Universidad de Costa Rica.

Ponder, W.F., Carter, G.A., Flemons, P., \& Chapman, R.R. (2001). Evaluation of museum collection data for use in biodiversity assessment. Conservation Biology, $15,648-657$.

Shaffer, H.B., Fisher, R.N., \& Davidson, C. (1998). The role of natural history collections in documenting species decline. Trends in Ecology and Evolution, $13,27-30$.

Solís-Marín FA, Alvarado, J.J., Abreu-Pérez, M., Aguilera, O., Alió, J., Bacallado-Aránega, J.J., Barraza, E., Benavides-Serrato, M., Benítez-Villalobos, F., Betancourt-Fernández, L., Borges, M., Brandt, M., Brogger, M.I., Borrero-Pérez, G.H., Buitrón-Sánchez, B.E., Campos, L.S., Cantera, J., Clemente, S., Cohen-Renjifo, M., Coppard, S., Costa-Lotufo, L.V., del Valle-García, R., Díaz, Y., Díaz de Vivar, M.E., Díaz-Martínez, J.P., Durán-González, A., Epherra, L., Escolar, M., Francisco, V., Freire, C.A., GarcíaArrarás, J.E., Gil, D.G., Guarderas, P., Hadel, V.F., Hearn, A., Hernández, J.C., Hernández-Delgado, E.A., Herrera-Moreno, A., Herrero-Pérezrul, M.D., Hooker, Y., Honey-Escandón, M.B.I., Lodeiros, C., Luzuriaga, M., Manso, C.L.C., Martín, A., Martinez, M.I., Martínez, S., Moro-Abad, L., Mutschke, E., Navarro, J.C., Neira, R., Noriega, N., Palleiro-Nayar, J.S., Pérez, A.F., Pérez-Ruzafa, A., Prieto-Rios, E., Reyes, J., Rodríguez, R., Rubilar, T., Sancho-Mejías, T., Sangil, C., Silva, J.R.M.C., Sonnenholzner, J.I., Ventura, C.R., Tablado, A., Tavares, Y., Tiago, C.G., Tuya, F., \& Williams, S.M. (2013). Appendix, p. 543654. In: Alvarado, J.J. \& Solís-Marín, F.A. (ed.). Echinoderm Research and Diversity in Latin America. Berlin: Springer.

Suarez, A.V. \& Tsutsui, N.D. (2004). The value of museum collections for research and society. BioScience, $54,66-74$. 
Verrill, A.E. (1867). Notes on the echinoderms of Panama and west coast of America, with description of new genera and species. Transactions of the Connecticut Academy of Arts and Sciences, 1(2), 251-322.

Wellington, G.M. (1974). An Ecological Description of the Marine and Associated Environments at Monumento Nacional Cahuita. San José: Subdirección de Parques Nacionales, Ministerio de Agricultura y Ganadería (unpublished).

Winker, K. (2004). Natural history museums in a postbiodiversity era. BioScience, 54, 455-459.

Ziesenhenne, F.C. (1940). New ophiurans of the Allan Hancock Pacific Expeditions. Allan Hancock Pacific Expedetion, 8(2), 9-59. 